\title{
Patient perceptions about virtual clinical consultations during current COVID-19 pandemic: A multi-city survey across India
} Deepa Elizabeth Mathew ${ }^{1}$, Vinay Bothra ${ }^{2}$, Shipra Mathur ${ }^{3}$, Cheri Mathews John ${ }^{4}$, Natasha Susan John ${ }^{5}$, Suneela Nayak $^{6}$, Sanjiv Kumar ${ }^{7}$

${ }^{1}$ Consultant pediatrician, Apollo Speciality Hospital, Vanagaram, Chennai; ${ }^{2}$ Health Policy Advisor, WHO Timor - Leste; ${ }^{3}$ Consultant pediatrician, Family Health Clinic, Gurgaon; ${ }^{4}$ Visiting consultant pediatrician, Apollo Speciality Hospital, Vanagaram, Chennai; ${ }^{5}$ Medical student, ESIC medical college, Chennai; ${ }^{6}$ Consultant pediatrician, Nayak clinic Children and Eye, Bengaluru; ${ }^{7}$ Ex-Dean IIHMR and Ex-ED National Health System Resource Centre.

\begin{tabular}{|c|c|c|c|c|c|c|c|c|}
\hline Abstract & Introduction & Methodology & $\underline{\text { Results }}$ & Conclusion & References & Citation & \multicolumn{2}{|c|}{ Tables / Figures } \\
\hline \multicolumn{9}{|c|}{ Corresponding Author } \\
\hline \multicolumn{8}{|c|}{$\begin{array}{l}\text { Dr Cheri Mathews John, Angels Speciality Clinic, AL-190, 1st street, 12th Main road, Anna Nagar, Chennai- } \\
600040 \\
\text { E Mail ID: Cherimjohn@gmail.com }\end{array}$} & 回评回 \\
\hline
\end{tabular}

\section{Citation}

Mathew DE, Bothra V, Mathur S, John CM, John NS, Nayak S, Kumar S. Patient perceptions about virtual clinical consultations during current COVID-19 pandemic: A multi-city survey across India. Indian J Comm Health. 2021;33(2):368372. https://doi.org/10.47203/IJCH.2021.v33i02.025

Source of Funding: Nil Conflict of Interest: None declared

\section{Article Cycle}

Received: 22/04/2021; Revision: 03/06/2021; Accepted: 19/06/2021; Published: 30/06/2021

This work is licensed under a Creative Commons Attribution 4.0 International License.

\section{Abstract}

Introduction: COVID-19 has prevented many patients from accessing health care through traditional face-to-face clinic visits. Consequently, online consultations have gained popularity. Aim: To explore patient perceptions regarding virtual consultations. Methods: A voluntary online survey using a mix of quantitative and qualitative questions was administered to patients across selected cities in India using a social media platform. Responses were used to explore the characteristics of users, perceived advantages and disadvantages of online consultations and patient satisfaction. Results: There were 679 respondents (M 52.4\%: F 47.6\%) that had consulted doctors online; $91.8 \%$ were from 8 major metro cities. Interestingly, over $80 \%$ had never sought online consultation before the COVID-19 pandemic. $46 \%$ consultations were via videocalls, $26 \%$ through WhatsApp and $21 \%$ via telephone calls. The main advantages of online consultations cited by patients included a lower risk of infection (78.8\%), reduced waiting time (56.8\%) and travel time (58.3\%). The main disadvantages included a lack of physical examination (73.4\%), a perception that this was not as satisfying as a face-to face consultation (37.9\%) and difficulty in communication (24.5\%). 78.6\% patients rated their online consultations as either good or very good. However, given the choice, almost two-thirds felt they would still prefer face-face consultations. Conclusion: High levels of satisfaction from this survey suggests that teleconsultation has the potential to become a complementary method to access clinical care even after restrictions from the pandemic cease. The disadvantages of online consultations could be mitigated through evolving technologies such as digital stethoscopes and improvement in communication tools.

\section{Keywords}

Online Consultation; Virtual Consultation; Teleconsultation; COVID-19

\section{Introduction}

The COVID-19 global crisis has severely affected the delivery of essential health services(1). One of the distinctive trends of this pandemic has been the exponential increase in the use of telemedicine as a complementary method to access clinical care. The Centers for Disease Control and Prevention (CDC) in the USA published a report estimating that $41 \%$ of U.S. adults had delayed or avoided medical care including urgent or emergency care $(12 \%)$ and routine care $(32 \%)$ because of concerns about contracting COVID-19(2). On 25 March, 2020, the Ministry of Health and Family Welfare (India) published guidelines to facilitate the use of telemedicine(1). While this shift was prompted by the public health and social measures introduced to reduce the risk of viral transmission during the pandemic, increased use of telemedicine as a means of accessing clinical care is likely to remain as one of the legacies of this global crisis. This virtual method of patient-provider 
interaction could, potentially, "feel different" (for both patients and clinicians) from the more traditional face-toface consultations.

\section{Aims \& Objectives}

1. To explore patient perceptions during virtual consultations with a clinical service provider;

2. To investigate the characteristics of online patientprovider consultations;

3. To explore the modality, referral pathway and patient perspectives for virtual consultations vis-à-vis face-toface consultations;

4. To examine the advantages and disadvantages of telemedicine (from a patient's perspective)

\section{Material \& Methods}

This mixed method quantitative and qualitative survey was conducted to capture primary data across eight metro cities in India, with a focus on the metropolises of Delhi, Mumbai, Chennai, Bengaluru, Jaipur, Gurugram, Hyderabad and Trivandrum. The survey covered nine months of the pandemic in India during the period April to December, 2020.

Potential respondents were identified using a purposively selected sample from established clinical, professional and social networks in these cities; 'snowballing' was used to supplement numbers from this initial sampling frame. The survey instrument consisted of a voluntary, anonymous self-report online questionnaire created in Google forms and administered to potential respondents through a popular social media platform (WhatsApp), sent out after 270 days of lock-down. The questionnaire collected demographic details of respondents and included 20 multiple-choice questions investigating various aspects of their experience of virtual consultations.

To assess the validity of the questionnaire, we reviewed validated questionnaires to elicit client feedback on health service delivery and adapted them to suit the needs of this specific study. This was piloted and tested through convenient rapid sampling before being rolled out to the participants in the study.

As the aim of this study was to determine patient's perceptions about tele-consultations, a target of 650 patients was set to get feedback from enough participants to be able to conduct a meaningful review. This was predicated by the time duration of the survey, the need for an initial rapid review and the effectiveness of the snowballing method to enroll the maximum number of respondents.

The data was collected, collated and analysed using a standard commercial software package. This was used to identify demographic characteristics of respondents, categorize barriers and facilitators and calculate rates and proportions for different patient perception related questions.

[Patient perceptions...] | Mathew DE et al

\section{Results}

Of the 1,600 people administered the survey, 679 responses were received - corresponding to a response rate of $42.4 \%$.

As for demographics, the gender breakdown showed a slight preponderance of men accessing virtual consultations $152.4 \%$ of men compared to $47.6 \%$ of women). The most common age-bracket for seeking this service was $50-60$ years (37.1\%). The vast majority of respondents (91.8\%) belonged to one of the 8 major cities. Although questions on income were not asked, this survey was targeted at an urban population with access to social media, it may be safe to presume that the majority of respondents were from the medium to higher socioeconomic quintiles.

523 of the 679 respondents (77\%) indicated that they had accessed virtual consultation(s) with a clinician at least once during the nine months of the pandemic. Of these, $72 \%$ opted for online consultation (Figure 1) on multiple occasions (2-3 consultations being more common - 52\% of all virtual encounters). Further questions on patient perceptions were only asked to those who had accessed one or more of these virtual consults.

One of the most remarkable, though not entirely surprising, findings from this survey was the number of people for whom this was the first time they have ever accessed a clinician using an online platform - this was over four out of every five virtual consultations (80.9\%).

The majority of respondents preferred to consult their own 'regular' doctor (62\%); $19 \%$ were referred to other clinicians through their network of family and friends; only $10 \%$ preferred to access a clinician using one of the popular commercial online clinical portals (Figure 2).

The most popular mode of consultation (Figure 3) was via a video call between provider and patient (46\%) followed by WhatsApp chat/text messaging (26\%) and phone calls (21\%).

Almost all respondents (92.9\%) felt they were given sufficient time by the clinician during the virtual consultation. $12.8 \%$ felt the need to visit a clinician faceto-face within a week of the online consult because the medical issue had not been resolved.

The major advantages cited by patients for choosing virtual over face-to-face consultation, in decreasing order of importance (Table 1), included: (1) fear of catching contagion at the doctor's clinic (78.8\%); (2) saving travel time/avoiding traffic (58.3\%); (3) avoiding long waiting times at the clinic $(56.8 \%)$; (4) ability to consult appropriate experts (44.9\%); (5) value for money (13.2\%). The main disadvantages highlighted in this survey (Table 2), in decreasing order, included: (1) reduced satisfaction due to inability to receive a physical examination (73.4\%); (2) lack of face-to-face contact/felt less 'connected' to the clinician (37.9\%); (3) inability to show past reports and 
prescriptions in a user-friendly manner (12.4\%) and; (4) did not consider it 'value for money' (4.6\%).

Even though over $78.6 \%$ of all respondents rated user satisfaction as excellent (44.4\%) or very good (34.2\%) it was significant that $63.3 \%$ said that they would still opt for a face-to-face consultation post COVID-19.

\section{Discussion}

The COVID-19 pandemic has accelerated teleconsultations as an almost disruptive technology that challenges the conventional method of face-to-face clinical consultations. For most of them (80\%), this crisis has offered them their first ever experience of a virtual consultation. While the majority of respondents, at least in this particular survey, demonstrated high levels of satisfaction, the fact that almost all of them still preferred a face-to-face consultation indicates that the weaknesses identified by respondents to this modality of patientclinician interaction will need to be addressed to ensure improved satisfaction and a better patient experience. This trend has the potential to pick up, especially, for mild/moderate cases as patient experience improves. Teleconsultations could also complement initial face-toface visits for follow up.

A primary concern raised in this survey was the inability to conduct a physical examination, a weakness inherent with this method of consultation. Others included a lack of awareness of telemedicine as an option, the safety and efficacy of telemedicine in the light of current circumstances, patient preferences for seeing their own doctor as opposed to someone with whom they have no established professional relationship and difficulty in discussing reports and test results $(3,4)$. Barriers like the inability to perform a physical exam(5) have been partially overcome by asking the patient to check their temperature via a home thermometer, observing a patient's general appearance via a video call, observations of a patient's respiration effort (including use of accessory respiratory muscles, effort of breathing, and speech), noticing the characteristics of the patient's cough (whether dry or productive), examination of the oropharynx via video, and measurement of blood pressure and pulse using home digital instruments and oxygen saturation using Oximeters. Improvements in emerging technologies such as more user-friendly, validated and reliable tools such as digital stethoscopes and wearable medical devices hold the promise of mitigating this deficit. This pandemic has increased virtual communication with patients $24 / 7$ via smartphones and webcam-enabled computers(6). Telemedicine visits may include video calls but also include SMS, email and mobilephone applications as well as patient worn devices and chatbots(7). In addition, enhancements to improve the audio-visual quality of the interaction is likely to reduce the gap between face-to-face and virtual consultations.
For telemedicine to exploit the advantages of reduced waiting times, travel time, traffic and risk of transmission of contagion, it will be important to improve communication channels in the virtual setting so that the inherent disadvantages compared to a face-to-face visit are felt less acutely. Telemedicine appointments may virtually eliminate travel, waiting times and inconvenience, especially for the elderly and disabled. The introduction of novel Artificial Intelligence (AI) techniques should be considered; emerging technologies such as the use of a short video clip taken on a smart phone to calculate a child's weight is one of many such emerging trends. More advanced methods of virtual communication are constantly emerging; these will help reduce, if not totally eliminate, the feeling of lack of 'connectivity' during virtual consults.

Alternate ways to 'store and forward' medical reports, digital health records and investigations that take up less band-width and can be transmitted more easily between patient and provider will allow clinicians to review the patient's recent investigations and past medical history easily and comprehensively. A notable disadvantage of telemedicine, as is the case with any new and evolving technology, is the lack of consumer awareness regarding access and cost of this service(7).

In order to offer a truly patient centered approach, indepth qualitative research not only to better understand patient's barriers to access but also potential solutions (from respondents themselves) will facilitate a more userfriendly patient experience.

India has the advantage of widespread mobile and broad band penetration, even in smaller towns, cities and increasingly in rural areas. Like other regions across the world, digital inequality in India is lesser in magnitude as compared to income inequality; more than 8 out of 10 people in developing countries own a mobile phone(8).This offers a unique opportunity for emerging low and middle income countries, including India, to harness the potential offered by this opportunity and increase access to clinical care, reduce indirect costs of care-seeking and 'leave no one behind' or more aptly in this case, 'leave no one offline'. The expansion of initiatives such as 'Digital India' and 'Bharat Net' that aim to connect smaller towns with fast broadband will add impetus to these developments.

In order to achieve this, it is also critical that the introduction of regulatory safeguards with checks and balances keeps pace with this rapidly evolving technology to ensure that the patient-provider interaction remains safe, secure and confidential. This has often lagged behind, unable to keep pace with the rapid evolution of this technology(9).

\section{Conclusion}

For most patients (80\%) this pandemic provided their first ever opportunity to access a virtual platform to seek 
clinical care. Telemedicine appointments may virtually eliminate travel, waiting times and inconvenience, reduces risk of infection especially for the elderly and disabled. The high level of satisfaction for online consults suggests that this modality of patient-provider interaction has the potential to be an increasingly popular intervention - even after the current pandemic. The future patient-doctor interactions are likely to be a mix of face to face (only when clinical examination by the doctor is required) and virtual consultations. As digital penetration improves, weaknesses identified in the current system of online consultations (e.g., inability to conduct physical examinations and inadequate communication) could be mitigated through evolving technologies such as the use of digital stethoscopes, novel Al technology and better communication tools (and behaviors). It also brings into focus the need for expanding digital penetration and ensuring that regulatory safeguards provide a safe, secure, convenient, and confidential experience, both for patients as well as providers.

\section{Recommendation}

Given the popularity of teleconsultation, it would be advisable for health policy planners to create systems to support and facilitate this modality. Weaknesses identified in the current system of online consultations (e.g., inability to conduct physical examinations and inadequate communication) need to be mitigated through evolving technologies such as the use of digital stethoscopes, novel Al technology and better communication tools.

\section{Limitation of the study}

- Being a largely urban, large city survey, patient perceptions across smaller lower tier cities and rural areas has not been explored in this study.

- The clinical service providers in this survey were medical doctors; consultations with other health workers were not part of this survey.

- The physical distancing restrictions imposed by the pandemic precluded a deep dive through Focus Group Discussions to investigate reasons for dissatisfaction and potential solutions - in more detail.
[Patient perceptions...] | Mathew DE et al

- This survey was conducted to explore contemporaneous or 'synchronous' online consultations between patient and provider. It did not explore non-synchronous methods such as 'store and forward' for radiology referrals; neither does it investigate provider-provider virtual contacts.

\section{Relevance of the study}

There has been increased use of telemedicine during the current COVID-19 pandemic. Our study reveals a high level of satisfaction with online consultations and identifies gaps to be addressed for it to remain a popular way of accessing healthcare by the public. With increased advancements in technology, there is the potential for this mode of consultation to flourish.

\section{Authors Contribution}

DEM: Concept, design, data acquisition, data analysis, finalization; SK: Manuscript editing and finalization; SM: data acquisition, analysis, manuscript editing and finalization; NSJ: Design, data acquisition, data analysis; SN: Design, data acquisition, data analysis and finalization; CMJ: Concept, design, data acquisition, analysis, manuscript drafting and finalization; VB: Design, data analysis, manuscript drafting and finalization

\section{References}

1. Ministry of Health and Family Welfare. https://www.mohfw.gov.in/pdf/Telemedicine.pdf

2. Centers for Disease Control and Prevention. https://www.cdc.gov/mmwr/volumes/69/wr/mm6936a4.htm

3. Portnoy J, Waller M, Elliott T. Telemedicine in the era of COVID-19. J Allergy ClinImmunolPract2020;8:1489-91.

4. Thirthalli J, Manjunatha N, Math SB. Unmask the mind! Importance of video consultations in psychiatry during COVID-19 pandemic Schizophr Res 2020;S0920-9964:30357-1.

5. Hollander JE, Carr BG. Virtually Perfect? Telemedicine for Covid-19. N Engl J Med. 2020 Apr 30;382(18):1679-1681. doi: 10.1056/NEJMp2003539. Epub 2020 Mar 11. PMID: 32160451

6. Keesara S, Jonas A, Schulman K. Covid-19 and Health Care's Digital Revolution. N Engl J Med. 2020 Jun 4;382(23):e82. doi: 10.1056/NEJMp2005835. Epub 2020 Apr 2. PMID: 32240581.

7. Telehealth satisfaction study: J.D. power, 2019. https://www.americantelemed.org/resources/telehealthadoption-and-usage/

8. World Development Report 2016: Digital dividends. Washington DC: World Bank. 2016

9. Operational framework for primary health care. Transforming vision into action. WHO. 2020

\section{Tables}

\section{TABLE 1 ADVANTAGES OF VIRTUAL CONSULTATIONS}

\begin{tabular}{|l|l|}
\hline Lesser risk of infection & $78.8 \%$ \\
\hline Saving travel time & $58.3 \%$ \\
\hline Ability to consult appropriate experts & $56.8 \%$ \\
\hline Value for money & $44.9 \%$ \\
\hline
\end{tabular}




\section{TABLE 2 DISADVANTAGES OF VIRTUAL CONSULTATIONS}

Lack of physical examination and laboratory tests

Not as satisfying as in person consultation

Inability to adequately convey the problem to the doctor

$24.5 \%$

Inability to show past reports

$12.4 \%$

No value for money

$4.6 \%$

\section{Figures}

FIGURE 1 NUMBER OF CONSULTATIONS DONE SINCE THE LOCK-DOWN STARTED

घ1 $\square 2$ to $3 \square 4$ to 5 घ6 ormore

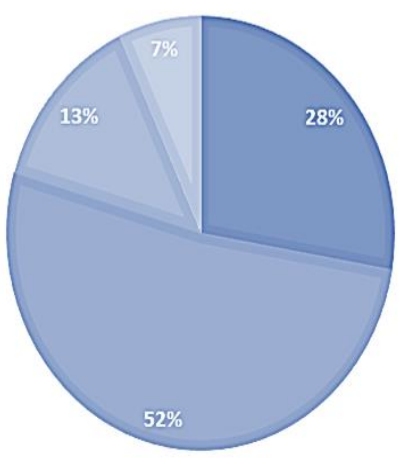

FIGURE 2 CHOOSING A DOCTOR ONLINE

-They are my regular doctor

aGoogle search

$\square$ Online portals like Practo/Docon/Mfine etc. $\square$ Hospital apps

aReferred by a friend/relative

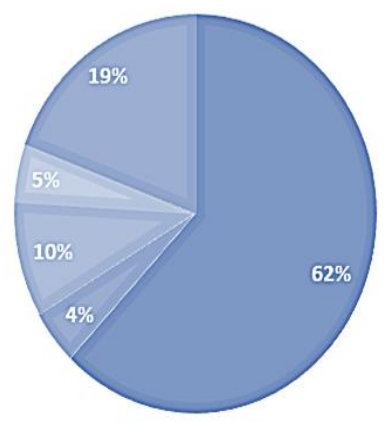

\section{FIGURE 3 MODE OF CONSULTATION}

a WhatsApp chatting/text messaging

a Phone call घ Video call

apps or websites

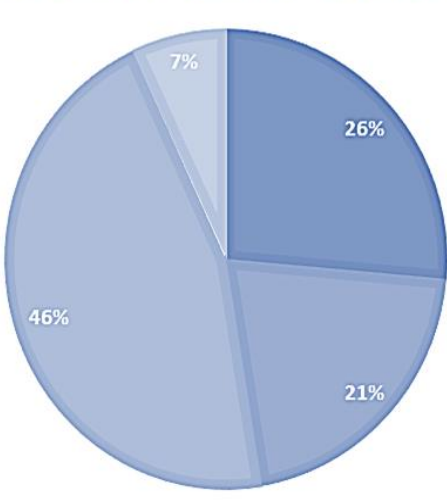

